03

\title{
Экспериментальное исследование охлаждения пластины электрическим ветром от игольчатого электрода
}

\author{
() И.А. Елагин, П.Ю. Марковский, Ю.К. Стишков \\ Санкт-Петербургский государственный университет, \\ 199034 Санкт-Петербург, Россия \\ e-mail: i.elagin@spbu.ru
}

Поступило в Редакцию 6 июня 2018 г.

В окончательной редакции 6 июня 2018 г.

Принято к публикации 28 октября 2019 г.

\begin{abstract}
Экспериментально исследовано охлаждение нагретой пластины, играющей роль заземленного электрода, с помощью узкой интенсивной струи электрического ветра от игольчатого коронирующего электрода, направленной перпендикулярно к центру пластины. При фиксированной средней температуре нагревателя получена зависимость отводимой с помощью ветра тепловой мощности от расходуемой на поддержание коронного разряда электрической мощности при различных межэлектродных расстояниях и напряжениях. Показано, что небольшая струя электрического ветра может эффективно охлаждать достаточно массивную пластину, отводя до $23 \mathrm{~W}$ тепловой мощности при затрачиваемой электрической мощности до $0.7 \mathrm{~W}$. Также проведено экспериментальное исследование структуры электрического ветра в рассматриваемой системе электродов. Для визуализации течений и получения распределений скорости использовалась установка лазерной анемометрии. Скорости в центральной струе электрического ветра достигали $8-9 \mathrm{~m} / \mathrm{s}$. При положительной полярности коронирующего электрода струя получается более узкой и быстрой. Однако эффективность охлаждения при положительной и отрицательной полярностях примерно одинакова.
\end{abstract}

Ключевые слова: коронный разряд, электрический ветер, охлаждение, лазерная анемометрия, электроды игла-плоскость.

DOI: $10.21883 / J T F .2020 .04 .49078 .227-18$

\section{Введение}

В чехле коронного разряда в воздухе образуются ионы, которые при перемещении во внешней зоне разряда передают свой импульс нейтральным молекулам, вызывая появление довольно интенсивных воздушных потоков [1], которые называются электрическим (или ионным) ветром. Направляя эти потоки на нагретые поверхности, можно существенно интенсифицировать отвод тепла с этих поверхностей $[2,3]$.

Использование электрического ветра для охлаждения имеет ряд преимуществ, таких как бесшумность, отсутствие трущихся частей, простота конструкции и т.п. Небольшие поперечные размеры образующихся потоков (порядка миллиметра) и высокая скорость течений (несколько метров в секунду) позволяют существенно уменьшить толщину теплового пограничного слоя у поверхности нагретого объекта, на который направлена струя. Из-за этих особенностей в последнее время делается много попыток применять электрический ветер для охлаждения миниатюрных объектов [3,4], так как обычные варианты охлаждения в большинстве своем на микромасштабах недостаточно эффективны.

В настоящей работе исследован электрический ветер средних масштабов, возникающий в системе электродов игла-плоскость. При этом плоская пластина, являющаяся также заземленным электродом, нагревалась до достаточно высокой температуры (порядка $360 \mathrm{~K}$ ), кото- рая близка к критической для многих конструктивных элементов различных технических устройств. В отличие от многих работ, например [5], в которых исследуется локальная интенсификация охлаждения тонкой струей электрического ветра, здесь рассматривается достаточно протяженная и массивная пластина. При этом струя, направленная от высоковольтного игольчатого электрода перпендикулярно к центру нагретой поверхности, позволяет эффективно охлаждать весь достаточно протяженный объект целиком, значительно увеличивая отвод тепла от его поверхности по сравнению с естественными условиями. Эффективность охлаждения существенно зависит от электрических параметров системы, основным при этом является затрачиваемая электрическая мощность, которая расходуется на поддержание коронного разряда. В настоящей работе рассмотрен широкий диапазон напряжений при различных полярностях игольчатого электрода, а также проведено варьирование величины межэлектродного расстояния.

Также необходимо отметить, что само по себе явление электрического ветра на данный момент изучено недостаточно подробно из-за его сложности и взаимосвязи различных физических процессов. Существует достаточно много работ, в которых экспериментально изучаются характеристики электрического ветра в различных системах электродов, например [6-9]. Однако зачастую им не хватает общности. Работ по исследованию структуры поля скоростей течений достаточно мало, что 
связано с их сложностью, отметим, например [8-10]. В последнее время с развитием новых методов стала возможна подробная визуализация течений, которая позволяет судить об особенностях конвективного переноса вещества, в том числе за счет электрического ветра. Однако стоит отметить тенденцию применения этих методов в данной области к системам многих электродов, когда возникающие струйные течения имеют достаточно сложный вид (что характерно, например, для электрофильтров [11]). При исследовании процесса охлаждения, особенно в микромасштабах, обычно структуру течений не исследуют вовсе [5], хотя именно она определяет интенсификацию отвода тепла. Некоторые данные о структуре течения может дать компьютерное моделирование, однако соответствующие расчеты сложны, в основном применяются различные упрощенные модели [12-15].

В настоящей работе для визуализации течений при электрическом ветре используется PIV-метод, который позволяет получать подробное распределение скоростей в различных сечениях. Приведены интегральные зависимости отводимой тепловой мощности при постоянном перегреве от затрачиваемой электрической мощности, а также локальное распределение температуры по поверхности пластины при ее охлаждении струей электрического ветра.

\section{1. Постановка эксперимента}

В настоящей работе исследуются возможности охлаждения алюминиевой пластины (размеры $190 \times 110 \times 6 \mathrm{~mm}$, рис. 1) с помощью электрического ветра в открытом воздушном пространстве. Рассматривается интенсивное охлаждение только с одной „лицевой“ стороны пластины, для этого она обратной стороной помещается в кожух из теплоизолирующего поролона толщиной $100 \mathrm{~mm}$. Пластина была расположена горизонтально лицевой стороной наверх. Игольчатый электрод располагался перпендикулярно пластине напротив центра ее лицевой стороны, как показано на рис. 1.

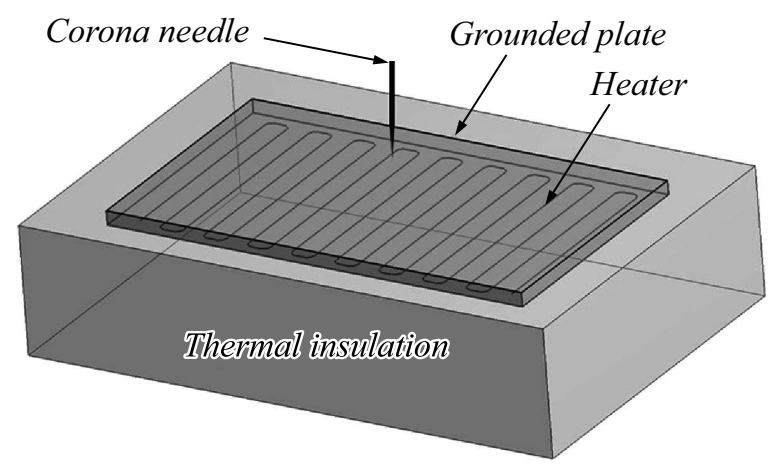

Рис. 1. Упрощенная схема основных элементов экспериментального макета.

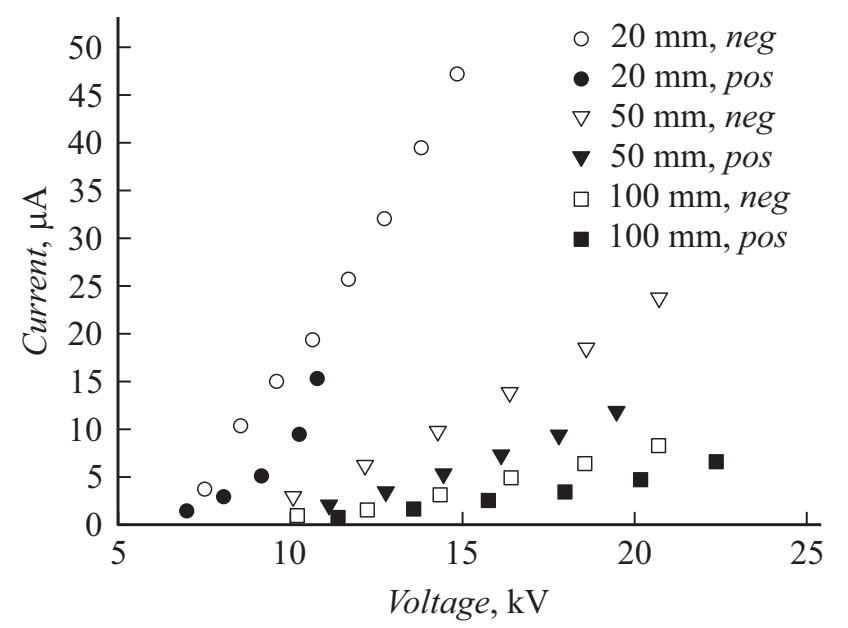

Рис. 2. $\mathrm{BAX}$ для различных межэлектродных расстояний при положительной и отрицательной полярностях игольчатого электрода.

Для нагрева пластины использовался нихромовый провод, равномерно намотанный на текстолитовую подложку, плотно прижатый к алюминиевой пластине с обратной стороны и подключенный к источнику напряжения. Эта система обеспечивала перегрев пластины в естественных условиях до $150 \mathrm{~K}$ относительно температуры окружающей среды. Для обеспечения равномерного отвода тепла за счет излучения, повторяемости результатов, а также для улучшения качества получаемых с помощью тепловизора (Fluke Ti32) данных о распределении температуры лицевая поверхность пластины была равномерно покрыта графитовой смазкой graphit 33, обеспечивающей сухое электропроводящее покрытие, и слоем аэрозоля, который использовался для визуализации течений с помощью PIV-установки. Коэффициент серости поверхности при этом равен 0.9 . Для контроля температуры пластины при установлении тепловых процессов, а также для градуировки тепловизора использовались терморезисторы, встроенные в пластину с обратной стороны. На иглу (рис. 1), диаметр кончика которой составлял примерно $100 \mu \mathrm{m}$, подавался высокий потенциал (положительной либо отрицательной полярности) с помощью высоковольтного источника постоянного напряжения ВИДН-30. Напряжения и токи в системе измерялись с помощью АЦП L-Card L-761.

В сечении, перпендикулярном пластине и проходящем через иглу, с помощью PIV-установки (FlowMaster от компании LaVision) снимались картины распределения скоростей. Для визуализации течений использовался аэрозоль $\mathrm{C}_{26} \mathrm{H}_{50} \mathrm{O}_{4}$, частички которого имеют малые размеры и практически не влияют на основные физические характеристики рассматриваемого явления [10]. Специальная обработка последовательности кадров позволяет получить распределение скоростей в плоскости съемки. Учитывая протяженность пластины, а также необходимость исследования достаточно 

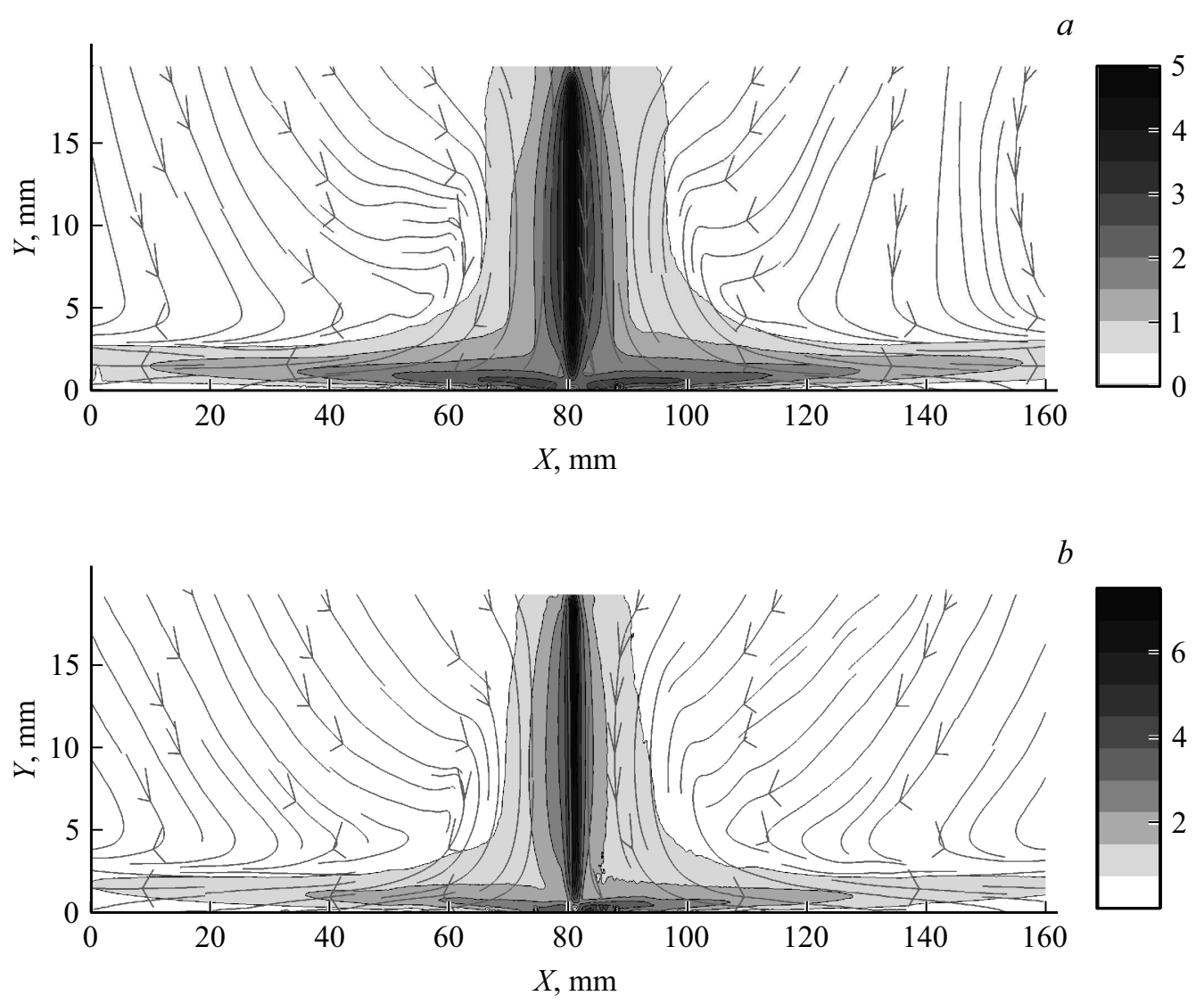

Рис. 3. Распределение скорости электрического ветра $(\mathrm{m} / \mathrm{s})$ при отрицательной $(a)$ и положительной $(b)$ полярностях.

больших межэлектродных расстояний, для детального исследования структуры воздушных течений производилась съемка нескольких участков в одной и той же плоскости сечения, а потом эти данные объединялись для получения высокого пространственного разрешения.

При проведении исследований пластина нагревалась до установившегося состояния. Температура в большинстве экспериментов по поверхности пластины распределена достаточно однородно из-за ее толщины и высокой теплопроводности. Характерное среднее значение перегрева $\Delta T=65 \mathrm{~K}$ для пластины фиксировалось для каждого эксперимента с помощью подбора необходимой мощности нагревательного элемента. При этом легче оценивать эффективность интенсификации охлаждения пластины за счет электрического ветра, так как от температуры параметры системы могут зависеть нелинейно, в частности коэффициент естественной конвекции. Также в этом случае остаются постоянными потери тепла, связанные с его возможным уходом за счет излучения, через поролон и другие элементы конструкции.

При проведении экспериментов с электрическим ветром расстояние между кончиком иглы и плоскостью варьировалось в диапазоне 20-100 mm. Напряжение изменялось в диапазоне от 5 до $25 \mathrm{kV}$.

\section{2. Результаты}

При увеличении напряжения на игольчатом электроде после некоторого порогового значения, определяемого геометрией системы и свойствами среды, у кончика иглы зажигается коронный разряд. Одновременно с возникновением разряда образуется электрический ветер, скорость которого определяется ионным током. На рис. 2 приводятся ВАХ коронного разряда в рассматриваемой системе электродов в широком диапазоне напряжений от порога зажигания до возникновения стримерной активности. При увеличении межэлектродного расстояния увеличивается пороговое напряжение зажигания разряда. Зависимости имеют характерную параболическую форму. При отрицательной полярности значения тока заметно выше. Величина разрядного тока от одиночной иголки невелика (несколько десятков микроампер), потребляемая разрядом электрическая мощность изменяется при этом в диапазоне $0-0.7 \mathrm{~W}$.

Распределение скорости электрического ветра, возникающего в рассматриваемой системе электродов при всех расстояниях между иглой и плоскостью, а также напряжениях, имеет характерный вид, представленный на рис. 3. Для отрицательной полярности приведен вариант при значениях напряжения и тока: $U=13.3 \mathrm{kV}, I=27.1 \mu \mathrm{A}$, для положительной поляр- 


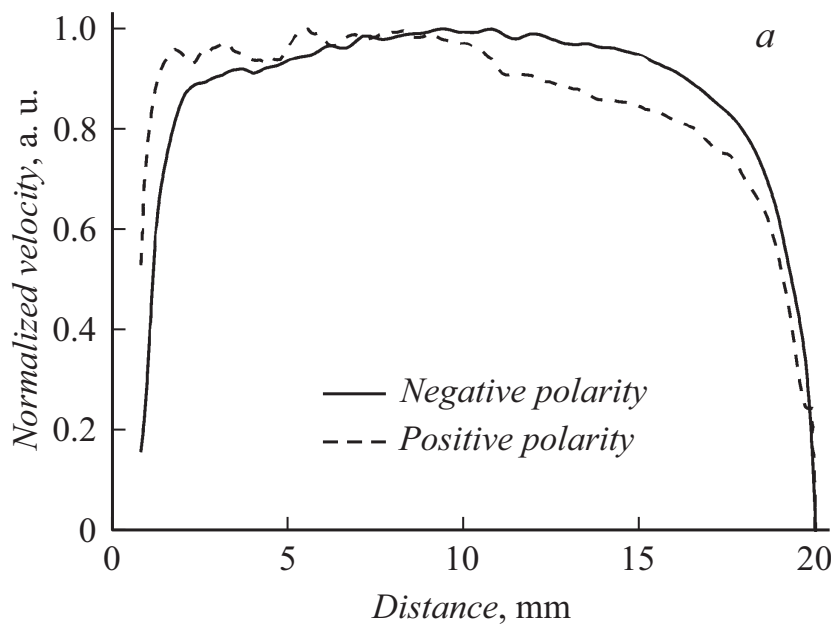

$b$

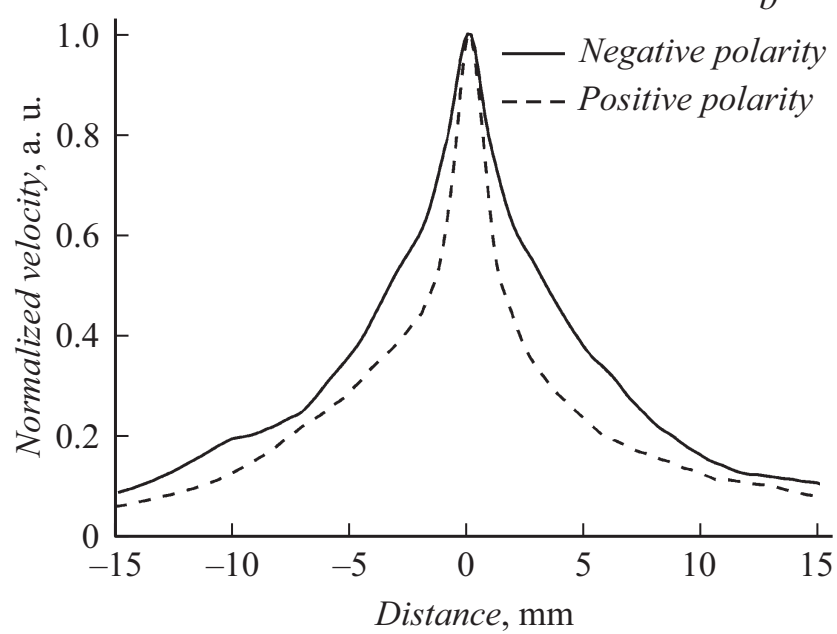

Рис. 4. Осевые распределения $(a)$ и профили $(b)$ скорости в центральной струе электрического ветра при отрицательной и положительной полярностях.

ности: $U=12.1 \mathrm{kV}, I=22.2 \mu \mathrm{A}$. Около кончика иглы формируется небольшая зона ускорения, в которой за счет действия кулоновской силы (определяемой электрическим полем и объемным зарядом) воздух быстро разгоняется, образуя узкую струю, направленную перпендикулярно к поверхности пластины. Максимальные значения скорости при этом достигают нескольких метров в секунду. После столкновения с пластиной струя радиально расходится в стороны и течет вдоль ее поверхности, при этом скорость в пограничном слое также достаточно высока (порядка $1 \mathrm{~m} / \mathrm{s}$ ). Если сравнить распределения скорости при отрицательной и положительной полярностях, то можно заметить, что в последнем случае струя более узкая и быстрая. Поток воздуха в центральной струе, определенный из экспериментальных данных с помощью интегрирования распределения скорости в центре струи с учетом осевой симметрии, примерно одинаковый при отрицательной и положительной полярностях $(0.3 \mathrm{~m} 3 / \mathrm{s})$.
На представленных на рис. 4 линейных графиках с нормированными осевыми (от иглы к пластине) и профильными (в центре струи) распределениями скорости можно более подробно проанализировать структуру центральной струи. Зона ускорения составляет примерно $2 \mathrm{~mm}$ как для отрицательной, так и для положительной полярностей (рис. $4, a$ ), что говорит об очень локальном распределении действующей на воздух объемной кулоновской силы, сосредоточенной в области максимального электрического поля у кончика иглы. Далее следует зона равномерного течения, высокая скорость на большей части межэлектродного промежутка поддерживается практически постоянной. Размеры зоны торможения у плоского электрода малы и составляют для обеих полярностей примерно $2-3 \mathrm{~mm}$. На профильных распределениях скорости, построенных по отрезку, перпендикулярному струе, в ее центре, можно отметить, что ширина струи для отрицательной полярности составляет примерно $6 \mathrm{~mm}$. Для положительной полярности эта величина меньше, она равна примерно $3 \mathrm{~mm}$. Компьютерное моделирование электрического ветра в униполярном приближении [16] показывает, что присутствие электронов в окрестности чехла коронного разряда приводит к расширению струи по сравнению с ситуацией, когда электроны не могут выходить за пределы чехла короны [17]. Чехол коронного разряда имеет малую толщину, порядка $10^{-4} \mathrm{~m}$ [18]. При положительной полярности электроны, возникающие в чехле, сразу направляются к электроду. При отрицательной полярности направление поля противоположное, и электроны распространяются на некоторое расстояние во внешнюю зону, лишь на расстоянии порядка $10^{-3} \mathrm{~m}$ превращаясь в отрицательные ионы в результате прилипания [17]. В связи с этим при положительной полярности струя электрического ветра тоньше, чем при отрицательной.

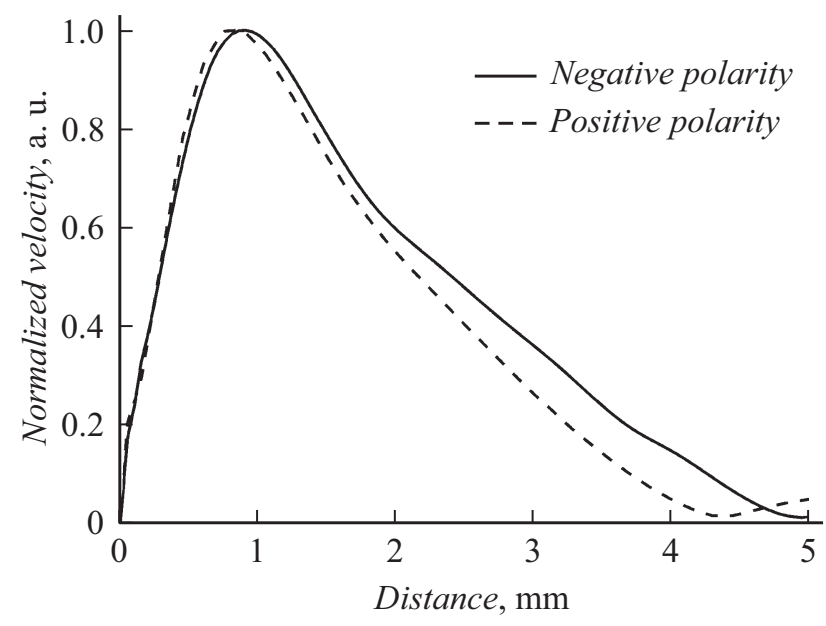

Рис. 5. Профили скорости в динамическом пограничном слое в боковой струе электрического ветра при отрицательной и положительной полярностях. 


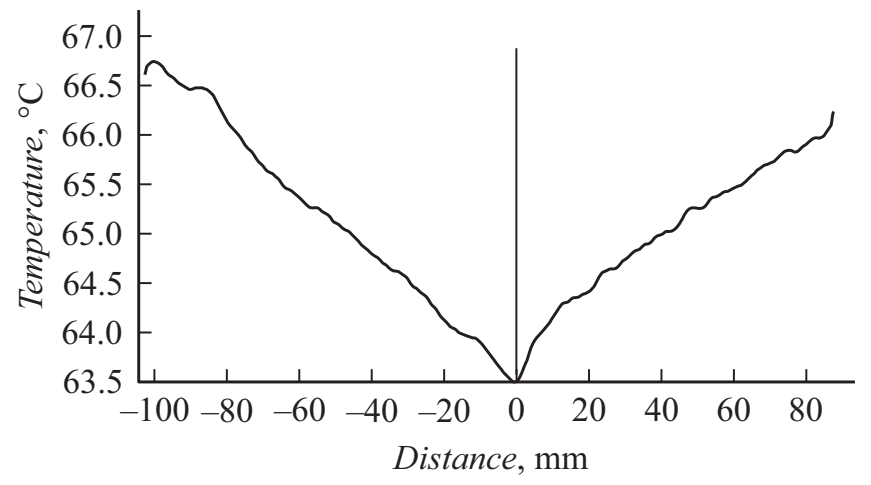

Рис. 6. Распределение перегрева вдоль поверхности пластины.

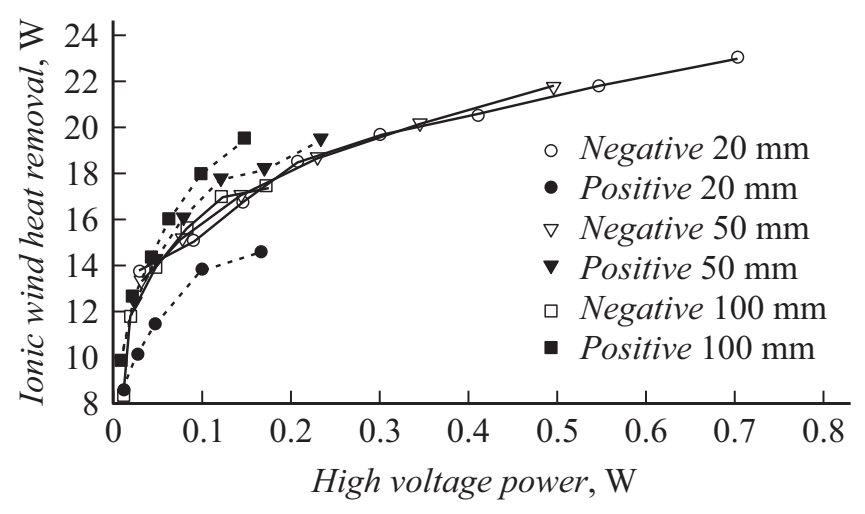

Рис. 7. Эффективность охлаждения пластины с помощью электрического ветра.

Максимальный отвод тепла с поверхности пластины, очевидно, находится в центре пластины, куда дует интенсивная струя электрического ветра, полностью разрушающая изначально достаточно широкий тепловой слой, характерный для естественной конвекции в отсутствии внешних вынужденных течений $[8,15]$. Однако пристеночная радиально расходящаяся струя ветра, линейное распределение профиля которой представлено на рис. 5 (по пути перпендикулярно пластине при $X=50 \mathrm{~cm}$ соответственно рис. 3), также приводит к значительной интенсификации теплоотвода в каждой точке поверхности нагретой пластины, так как она имеет достаточно высокую скорость. Кроме того, максимум скорости располагается очень близко к поверхности пластины, на расстоянии примерно $1 \mathrm{~mm}$ (рис. 5), что значительно меньше, чем в естественных условиях при любом расположении плоского нагревателя как горизонтальном, так и вертикальном $[8,15]$.

Описанные выше эффекты, а также достаточно большая толщина пластины, приводят к тому, что перепад температуры вдоль пластины небольшой, он составляет всего несколько градусов (рис. 6, приведен пример для отрицательной полярности, $U=16 \mathrm{kV}$ ). Поэтому вся пластина в целом охлаждается достаточно эффективно с помощью интенсивной тонкой струи электрического ветра.

На рис. 7 представлены зависимости отводимой со всей пластины тепловой мощности с помощью электрического ветра, от электрической мощности коронного разряда (при фиксированном среднем перегреве пластины $\Delta T=65 \mathrm{~K})$. Приведены данные для напряжений разных полярностей, а также для различных межэлектродных расстояний. Наибольшая эффективность охлаждения (можно определить как отношение отводимой тепловой мощности к мощности коронного разряда) с помощью электрического ветра наблюдается на начальных участках кривых. Отводимая тепловая мощность достигает $23 \mathrm{~W}$, в то время как электрическая мощность не превышает $0.7 \mathrm{~W}$. C увеличением напряжения на игле эффективность охлаждения несколько снижается. Здесь также необходимо отметить, что в естественных условиях теплоотдача для рассматриваемой горизонтально расположенной пластины составляет примерно $5 \mathrm{~W}[8]$. То есть с помощью электрического ветра от одиночного игольчатого электрода удалось существенно превысить эту величину. Также на графиках можно отметить общий характер зависимостей для всех межэлектродных расстояний.

\section{Заключение}

В работе приведены результаты экспериментального исследования влияния электрического ветра от одиночного игольчатого электрода на теплоотвод от нагреваемой поверхности.

С помощью установки лазерной анемометрии исследованы поля скоростей электрического ветра в системе электродов игла-плоскость в широком диапазоне напряжений при различных межэлектродных расстояниях для положительной и отрицательной полярностей.

Скорости электрического ветра зависят от напряжения, тока, полярности и межэлектродного расстояния. В общем случае от иглы образуется тонкая осесимметричная струя ветра, направленная от кончика иглы к центру пластины. Максимальная скорость в ней достигает $8-9 \mathrm{~m} / \mathrm{s}$. При положительной полярности струя получается более узкая и быстрая, чем при отрицательной.

Использование одиночной осесимметричной струи электрического ветра для охлаждения достаточно массивной пластины оказывается весьма эффективным, что связано с высокой скоростью ветра в центральной струе и в боковых струях, омывающих поверхность пластины. Зависимость отводимой за счет электрического ветра конвективной тепловой мощности от электрической мощности коронного разряда во всех случаях имеет схожий вид. При этом максимальная эффективность отведения тепла наблюдается на начальных участках кривых с максимальным градиентом. 


\section{Благодарности}

Исследования выполнены с использованием оборудования ресурсных центров „Геомодель“ и „Оптические и лазерные методы исследования вещества“ научного парка СПбГУ.

\section{Конфликт интересов}

Авторы заявляют, что у них нет конфликта интересов.

\section{Список литературы}

[1] Верещагин И.П. Коронный разряд в аппаратах электронно-ионной технологии. М.: Энергоатомиздат, 1985. $160 \mathrm{c}$.

[2] Bologa M.K., Grosu F.P. // Surf. Engineer. Appl. Electrochem. 2012. Vol. 48. N 5. P. 456-464.

[3] Wang H.-C., Jewell-Larsen N.E., Mamishev A.V. // Appl. Thermal Engineer. 2013. Vol. 20. N 1-2. P. 190-211.

[4] Jewell-Larsen N.E., Ran H., Zhang Y., Schwiebert M.K., Honer K.A. // Annual IEEE Semicond. Thermal Measurement and Management Symposium. 2009. P. 261-266.

[5] Chen I.Y., Guo M.-Z., Yang K.-S., Wang C.-C. // Intern. J. Heat and Mass Transfer. 2013. Vol. 57. N 1. P. 285-291.

[6] Moreau E. // J. Phys. D: Appl. Phys. 2007. Vol. 40. N 3. P. 605-636.

[7] Tsubone H., Ueno J., Komeili B., Minami S., Harvel G.D., Urashima K., Ching C.Y., Chang J.S. // J. Electrostatics. 2008. Vol. 66. N 1-2. P. 115-121.

[8] Елагин И.А., Яковлев В.В., Ашихмин И.А., Стишков Ю.К. // ЖТФ. 2016. Т. 86. Вып. 8. С. 95-101.

[9] Елагин И.А., Бегаль Д.И., Ашихмин И.А., Стишков Ю.К. // Письма в ЖТФ. 2017. Т. 43. Вып. 2. С. 24-30.

[10] Ashikhmin I., Stishkov Y.K., Yakovlev V. // Intern. J. Plasma Environ. Sci. Technol. 2015. Vol. 9. N 1. P. 13-17.

[11] Niewulis A., Podlński J., Berendt A., Mizeraczyk J. // Intern. J. Plasma Environ. Sci. Technol. 2014. Vol. 8. N 1. P. 60-71.

[12] Samusenko A., Stishkov Y., Zhidkova P. // Intern. J. Plasma Environ. Sci. Technol. 2015. Vol. 9. N 1. P. 24-28.

[13] Adamiak K. // J. Electrostatics. 2013. Vol. 71. N 4. P. 673-680.

[14] Елагин И.А., Стишков Ю.К. // ЖТФ. 2005. Т. 75. Вып. 9. C. $15-19$.

[15] Elagin I.A., Ashikhmin I.A., Samusenko A.V., Stishkov Y.K., Yakovlev V.V. // Intern. Conf. Dielectr. 2016. P. 151-154.

[16] Ашихмин И.А., Самусенко А.В., Стишков Ю.К., Яковлев В.В. // ЖТФ. 2015. Т. 85. Вып. 11. С. 65-72.

[17] Zhidkova P.S., Samusenko A.V. // Surf. Engineer. Appl. Electrochem. 2016. Vol. 52. N 4. P. 370-379.

[18] Zubkov T.N., Samusenko A.V., Stishkov Y.K. // Surf. Engineer. Appl. Electrochem. 2013. Vol. 49. N 6. P. 474-479. 